\title{
Duration of treatment with oral rehydration salts for vasovagal syncope in children and adolescents
}

\author{
Chuan Wen $^{1 \odot}$, Shuo Wang ${ }^{2,3 \odot}$, Runmei Zou ${ }^{1 \odot}$, Yuwen Wang ${ }^{1 \odot}$, Chuanmei Tan ${ }^{2,3 \odot, ~}$ \\ $\mathrm{Yi} \mathrm{Xu}^{1 \odot}$, Cheng Wang ${ }^{1 \odot}$ \\ ${ }^{1}$ Department of Pediatric Cardiovasology, Children's Medical Center, The Second Xiangya Hospital, Central South University \\ / Institute of Pediatrics, Central South University, Changsha 410011, Hunan, China; ${ }^{2} J i s h o u$ University School of Medicine, \\ Jishou,416000, China; ' Department of Pediatrics, The First People's Hospital of Changde City, Changde, Hunan, 415003, China.
}

\begin{abstract}
Background. Oral rehydration salt (ORS) is a first-line medication for vasovagal syncope (VVS) in children and adolescents. We retrospectively investigated the treatment with ORS-I (Na $90 \mathrm{mmol} / \mathrm{L}$ ) for VVS in children and adolescents to define appropriate duration of treatment.
\end{abstract}

Methods. All patients with a diagnosis of VVS, based on the first head-up tilt test (HUTT) response, and who accepted ORS-I treatment were enrolled. ORS was stopped when the HUTT response turned negative. Patients were followed for six months after cessation of ORS treatment.

Results. The study group included 129 patients ( 57 male, 72 female; mean age, $11.8 \pm 2.0$ years, age range, 7.017.0 years). Median duration of VVS was 4 months (range, 1 week to $>10$ years). The number of syncope ranged from 2 times to $>20$ times. Mean follow-up time was 27.8 \pm 6.9 weeks (range, 26-33 weeks). It took to 2 13 weeks for HUTT response to turn negative, with an average time of 8.4 weeks (95\% confidence interval, 6.89 9.84 weeks). There was no statistical difference for the time to negative HUTT response according to age groups ( $<12$-year-old vs. $\geq 12$-year-old), syncope type (vasodepressor vs. mixed), and the syncope frequency. No patient experienced syncope after cessation of ORS treatment.

Conclusions. Our findings suggest that ORS-I is an effective measure to treat children and adolescents with VVS. We recommend a treatment course of 2 months.

Key words: oral rehydration salts, vasovagal syncope, medication course, children, adolescents.

Vasovagal syncope (VVS) is the most common type of unexplained syncope in children and adolescents. Oral rehydration salts (ORS) is one of the first-line non-pharmacological measures. Increasing dietary intake of salt is the main measure of rehydration, ${ }^{1}$ however, clinically, it involves problems such as intake of water and salt is hard to control and their excessive intake can affect blood pressure. ORS have been used for more than 40 years in the prevention and treatment of mild dehydration caused by acute or chronic diarrhea. As an alternative measure

$\triangle$ Cheng Wang

wangcheng2nd@csu.edu.cn

Received 13th November 2019, accepted 26th January 2020. to increase dietary salt intake, ORS has been increasingly used in clinics. ${ }^{2-5}$ However, there is as yet no report on the medication course of ORS both at home and abroad. This paper aims to retrospectively analyze the clinical data of the children and adolescents with confirmed VVS in our hospital and discuss the medication course of ORS for VVS.

\section{Material and Methods}

Between March 2006 to October 2013, a total of 129 children and adolescents ( 57 males and 72 females; mean age, $11.8 \pm 2.0$ years; range, 7.0-17.0 years) with unexplained syncope for a course of 1 week to $>10$ years (median course of 4 months) and syncope episodes of 2 to $>20$ times 
(3.5 \pm 3.3 times on average; $\geq 2$ episodes in half a year) who visited Pediatric Syncope Clinic in the Second Xiangya Hospital of Central South University, China were enrolled. The inclusion criteria included the following: 1) other syncope diseases were ruled out on routine examination; 2) initial head-up tilt test (HUTT) response was positive and ORS therapy was given once VVS (including vasodepressor type and mixed type, excluding cardioinhibitory type) was confirmed. Patients whose diseases were failed to be confirmed on detailed inquiry of medical history, physical examination, biochemical examination, routine 12-lead ECG, 24-hour dynamic ECG, 24-hour ambulatory blood pressure, EEG, and skull CT or MRI, etc. were excluded. Patients were classified according to age ( $<12$-year-old and $\geq 12$-year-old), frequency of syncope (2-5 episodes and $\geq 6$ episodes). The patients were followed-up at 2-4 weeks as directed by medical order for collection of detailed medical history including recurrence of syncope after treatment, patients' compliance with ORS treatment etc. All patients showed good compliance with ORS, and HUTT was performed in every follow-up until negative HUTT response was shown. Syncope recurred no more during 6-month follow-up after ORS withdrawal. ${ }^{6-8}$

The informed consent was obtained from all the subjects directly or their guardians prior to enrollment. The study protocol (no. 2014012) was approved by the Ethics Committee of the Second Xiangya Hospital, Central South University, China.

Head-up tilt test (HUTT) was used for assessing patients with suspected syncope but lack of confirmed diagnosis after an initial assessment, or a differential diagnosis of convulsive syncope from epilepsy or pseudo-syncope from VVS. The head-up tilt period was performed under two conditions: passive (without any provocative drugs), and pharmacological (with drug challenge). Subjects were tilted at $60^{\circ}$ head upward, heart rate (HR), blood pressure (BP) and ECG were recorded continuously until either $45 \mathrm{~min}$. duration, or development of syncope or intolerable near syncope symptoms. If syncope occurred, patients were rapidly placed in the supine position. If syncope or presyncope did not occur, tilted posture was maintained, subjects were medicated with adjunctive agents, such as isoproterenol, nitrates, and clomipramine, and HR, BP and ECG were recorded for $20 \mathrm{~min}$. or syncope or presyncope occurred.

Syncope or pre-syncope symptoms accompanied with one of the following conditions during HUTT was defined as VVS: (1) $\mathrm{BP}<80 / 50 \mathrm{mmHg}(10.66 / 6.67 \mathrm{kPa})$ or $>25 \%$ reduction of mean $\mathrm{BP}$ relative to baseline $\mathrm{BP}$; (2) HR $<75$ bpm for children aged 4-6 years, $<65$ bpm for those aged 6-8 years , $<60 \mathrm{bpm}$ for those aged above 8 years; (3) ECG showing sinus arrest or premature junctional contractions; (4) atrioventricular block, asystole up to $3 \mathrm{sec}$. VVS was classified as: vasodepressor type (significant reduction in $\mathrm{BP}$ but insignificant change in $\mathrm{HR}$ ), cardioinhibitory type (significant reduction in HR but insignificant change in BP), or mixed type (significant reduction both in $\mathrm{BP}$ and HR). $\cdot{ }^{9,10}$

\section{Therapeutic regimen}

Patients who have been diagnosed as having VVS by HUTT were treated with ORS-I [Drug specification: $14.75 \mathrm{~g} / \mathrm{bag}$; composition: glucose of $11.00 \mathrm{~g}$, sodium chloride of $1.75 \mathrm{~g}$, potassium chloride of $0.75 \mathrm{~g}$, sodium bicarbonate of $1.25 \mathrm{~g}$, and osmotic pressure of $311 \mathrm{mOsm} / \mathrm{L}$, sodium 90 mmol/L; Manufacturer: Fuzhou Haiwangfuyao Pharmaceutical Co., Ltd. (Approval Number: H35021107)]. Dosage \& Administration: All the subjects were administered with ORS-I (14.75 g) that dissolved in $500 \mathrm{ml}$ (per day) of warm water or cold water in divided oral doses. ORS discontinued when negative HUTT response was shown. 


\section{Evaluation on curative efficacy}

The curative efficacy was evaluated based on clinical syncope episodes: Improvement was defined as decrease in syncope episodes or even no reoccurrence of syncope after treatment; no improvement was defined as no change or even increase in syncope episodes as compared with that before treatment.

\section{Statistical analysis}

The data were analyzed using SPSS17.0 software, with measurement data expressed as mean \pm standard deviation and counting data expressed as rate. Variance analysis was carried out for group comparison. A p-value $<0.05$ was considered statistically significant.

\section{Results}

\section{Curative efficacy}

When negative HUTT was shown in 129 VVS cases during follow-up, syncope reoccurred no more in 110 and significantly less frequent in 19 than before medication, with clinical response rate of $100 \%$. Negative HUTT response was noted at 2-13 weeks (8.4 weeks on average, 95\% confidence interval: 6.89-9.84).
Time to achieve negative HUTT response according to age groups and different hemodynamic types

No significant differences were noted in the time to achieve negative HUTT response between the $<12$-year-old group and $\geq 12$-year-old group $(p=0.068)$, or between vasodepressor type and mixed type $(p=0.218)$, and the interactions between age and the time to negative HUTT response or between hemodynamic types and the time to negative HUTT response were nonsignificant $(p=0.944)$ (Table I).

Time to achieve negative HUTT response according to age groups and frequency of syncope episodes

No significant differences were noted in the time to achieve negative HUTT response between the $2-5$ syncope episodes group and $\geq 6$ syncope episodes group $(\mathrm{p}=0.212)$. The interactions between age and syncope episodes were nonsignificant $(\mathrm{p}=0.115)$ (Table II).

\section{Discussion}

VVS is a condition that is marked by sudden loss of consciousness due to transient insufficient cerebral blood flow resulting from

Table I. Vasovagal syncope reaction type according to age groups; time to negative head-up tilt test response according to vasovagal syncope reaction type and age groups.

\begin{tabular}{lcccc}
\hline \multirow{2}{*}{ Age groups } & \multicolumn{2}{c}{ VVS reaction type, $\mathrm{n}(\%)$} & \multicolumn{2}{c}{$\begin{array}{c}\text { Time to negative HUTT response according to VVS } \\
\text { reaction type, weeks }\end{array}$} \\
\cline { 2 - 5 } & Vasodepressor type & Mixed type & Vasodepressor type & Mixed type \\
\hline$<12$ years $(\mathrm{n}=48)$ & $37(77)$ & $11(23)$ & $5.9 \pm 3.7$ & $8.1 \pm 5.6$ \\
$\geq 12$ years $(\mathrm{n}=81)$ & $56(69)$ & $25(31)$ & $8.9 \pm 7.0$ & $10.1 \pm 8.7$ \\
\hline
\end{tabular}

HUTT: head-up tilt test, VVS: vasovagal syncope

Table II. Frequency of syncope according to age groups; and time to negative head-up tilt test response according to frequency of syncope and age groups.

\begin{tabular}{lcccc}
\hline \multirow{2}{*}{ Age groups } & \multicolumn{2}{c}{ Frequency of syncope, $\mathrm{n}(\%)$} & \multicolumn{2}{c}{$\begin{array}{c}\text { Time to negative HUTT response according to } \\
\text { frequency of syncope, weeks }\end{array}$} \\
\cline { 2 - 5 } & 2 to 5 episodes & $\geq 6$ episodes & 2 to 5 episodes & $\geq 6$ episodes \\
\hline$<12$ years $(\mathrm{n}=48)$ & $44(92)$ & $4(8)$ & $6.2 \pm 4.2$ & $5.0 \pm 1.0$ \\
$\geq 12$ years $(\mathrm{n}=81)$ & $70(86)$ & $11(14)$ & $8.3 \pm 6.5$ & $9.0 \pm 7.1$ \\
\hline
\end{tabular}

HUTT: head-up tilt test 
diffident factors and fainting due to inability to maintain muscle tone. Studies have shown that Bezold-Jarisch reflex, hypovolemia and baroreflex dysfunction might be involved in the pathogenesis of VVS. Although VVS is self-limited with favorable prognosis, it causes recurrent syncope in $70 \%$ of pediatric and adolescent cases, and can even lead to syncoperelated somatic accidental injury thus affecting their physical and mental health, study and life. Therefore, VVS in children and adolescents still need intervention in an effort to reduce syncope episodes so as to improve their quality of life..$^{11,12}$

Non-pharmacological approaches are the firstline intervention measures in the management of VVS, aiming at increasing circulating blood volume, enhancing autonomic neuroregulatory function, and increasing orthostatic tolerance etc. Increasing intake of water and salt is the basic measure of non-pharmaceutical treatment. According to the guidelines for the diagnosis and management of syncope, expanding blood volume by increasing water and salt intake is the key to the management of VVS and is thus recommended to be one of the basic measures for VVS treatment. ${ }^{1,3,4}$

In the study of rehydration and salt supplementation for unexplained syncope, Cooper et al. ${ }^{13}$ reported symptom relief and significant increases in orthostatic tolerance and baroreceptor sensitivity in 178 adult patients with syncope or threatened syncope after oral administration with sodium chloride $100 \mathrm{mmol} /$ day $(5.85 \mathrm{~g})$ for 3 months. El-Sayed et al. ${ }^{14}$ made a study on 20 syncope patients (40-60 years) by treating the patients with sodium chloride 120 $\mathrm{mmol} /$ day for 8 weeks and noted a significant increase in plasma and blood volumes (100-500 $\mathrm{ml})$ in $80 \%$ patients and increased orthostatic tolerance in all, much as in those whose 24hour urinary sodium excretion was below 170 $\mathrm{mmol} /$ day. Mtinangi et al. ${ }^{15}$ found increase in orthostatic tolerance within 3 days after salt loading. Shichiri et al. ${ }^{16}$ reported increase in blood volume and significant increase in orthostatic tolerance in a 14-year-old orthostatic hypotension child after 48-hour treatment with sodium chloride at a dosage of $3 \mathrm{~g} /$ day, b.i.d. In addition, studies have shown that supplementation of salt and fluid is also effective for syncope in children and the elderly. ${ }^{17,18}$ Bellard et al. ${ }^{19}$ reported that increasing hydration alone does not improve orthostatic tolerance in patients with neurocardiogenic syncope (NMS). Wieling et al. ${ }^{20}$ suggested that adult patients with orthostatic syncope should increase their salt intake by $\geq 1 \mathrm{~g} /$ time (three times a day) and fluid intake by 2-2.5 liter per day. Guzman et al. ${ }^{21}$ pointed out that in the absence of contraindication, symptomatic patients should be encouraged to increase their salt and fluid intake to at least $2 \mathrm{~g} /$ day of sodium and 2 to $3 \mathrm{~L} /$ day of water, which is probably a cost-effective and safe strategy that should be used as firstline therapy. The study made by Claydon et $\mathrm{al}^{22}$ on the effect of salt supplementation on syncope patients by using HUTT and Doppler ultrasound showed significant improvement in symptoms, orthostatic tolerance and cerebral autoregulation, yet non-significant change in resting heart rate or blood pressure. The underlying mechanism might be the increase in orthostatic tolerance following increase in salt loading, which mightbeassociated to theincrease of plasma volume as well as the enhancement of sympathetic control on peripheral vascular system and the improvement of cerebral vascular autoregulation. The increase in responsiveness of vascular resistance during orthostatic period may be related to the increased baroreceptor sensitivity resulting from salt supplementation, which might pose a direct effect on vascular resistance in that it has been shown in some animal experiments that increasing salt intake could lead to enhanced response of isolated blood vessels to norepinephrine. ${ }^{23}$ However, the mechanism underlying the improved cerebral autoregulation remains unclear, with the only finding that the cerebral blood flow velocity depends less on blood pressure after salt load increase.

The curative efficacy of ORS for VVS has gained general consensus, and it has been recommended as one of the basic measures 
for the treatment of VVS by the guidelines for the diagnosis and management of syncope. In traditional therapy, increasing dietary salt intake is the main measure of ORS, yet it poses the risk of increasing blood pressure and has its limitations including changing the taste of food thus affecting food intake. Accurate control of water and salt intake can effectively reduce possible complications. ORS as an alternative to increase dietary salt intake has been gradually applied in clinics. However, there is yet no report on the medication course of ORS. At present, ORS has three generations (I, II, and III), and they were ranked in descending order in terms of osmotic pressures. ORS-I with a cheap price has been widely used in clinics since the 1970s when it was recommended by the World Health Organization (WHO) for clinical prevention and management of mild dehydration induced by acute or chronic diarrhea. In this study, ORS-I was selected for treating VVS in children and adolescents, with clinical syncope recurrence episodes as the criteria for evaluation of clinical symptom improvement and HUTT negative conversion as the end point to analyze the medication course of ORS for VVS. Our study on 129 children and adolescents with VVS showed that when negative HUTT was achieved after treatment with ORS therapy, syncope occurred no more in 110 cases, significantly less frequent in 19 than before treatment, with a response rate of $100 \%$. HUTT converted to negative at 2-13 weeks (8.4 weeks on average, with $95 \%$ confidence interval of 6.89 - 9.84).

No significant differences were noted in the time to achieve negative HUTT response between the $<12$ years old group and $\geq 12$ years old group, between the vasodepressor type and mixed type, or between the 1 syncope episode group and $\geq 6$ syncope episodes group, and the effects of their interactions on the time to achieve negative HUTT response were all nonsignificant.

In short, ORS is an effective therapy for VVS in children and adolescents. It is advisable to adjust the medication course of ORS for VVS in children and adolescents in accordance with the improvement of clinical symptoms and HUTT results. The recommended course of treatment for ORS is 2 months.

\section{REFERENCES}

1. Moya A, Sutton R, Ammirati F, et al; Task Force for the Diagnosis and Management of Syncope; European Society of Cardiology (ESC); European Heart Rhythm Association (EHRA); Heart Failure Association (HFA); Heart Rhythm Society (HRS); Guidelines for the diagnosis and management of syncope (version 2009). Eur Heart J 2009; 30: 26312671.

2. Chen L, Wang L, Sun J, et al. Midodrinehydrochloride is effective in the treatment of children with postural orthostatic tachycardia syndrome. Circ J 2011; 75: 927-931.

3. Chu W, Wang C, Wu L, Lin P, Li F, Zou R. Oral rehydration salts: an effective choice for the treatment of children with vasovagal syncope. Pediatr Cardiol 2015; 36: 867-872.

4. Zhang W, Zou R, Wu L, et al. The changes of electrolytes in serum and urine in children with neurally mediated syncope cured by oral rehydration salts. Int J Cardiol 2017; 233: 125-129.

5. Li W, Wang S, Liu X, Zou R, Tan C, Wang C. Assessment of efficacy of oral rehydration salts in children with neurally mediated syncope of different hemodynamic patterns. J Child Neurol 2019; 34: 5-10.

6. Liao D, Xu Y, Zou R, et al. The circadian rhythm of syncopal episodes in patients with neurally mediated syncope. Int J Cardiol 2016; 215: 186-192.

7. Zou R, Wang S, Zhu L, et al. Calgary Score and modified Calgary Score in the differential diagnosis between neurally mediated syncope and epilepsy in children. Neurol Sci 2017; 38: 143-149.

8. Zhang W, Wang S, Wang C, et al. Head-up tilt test results in child twins with nervous mediated syncope. Int J Cardiol 2016; 221: 194-197.

9. Wang C, Li Y, Liao Y, et al. 2018 Chinese Pediatric Cardiology Society (CPCS) guideline for diagnosis and treatment of syncope in children and adolescents. Sci Bull 2018; 63: 1558-1564.

10. Tao C, Liu X, Zhang C, ChenY, HuangY. Comments on 2018 CPCS guideline for diagnosis and treatment of syncope in children and adolescents. Sci Bull 2019; 64: 291-292.

11. Xu W, Wang T. Diagnosis and treatment of syncope in pediatric patients: a new guideline. Sci Bull 2019; 64: 357-358. 
12. Stewart JM. A new guideline for diagnosis and treatment of syncope in children and adolescents that stimulates further thought and discussion. Sci Bull 2018; 63: 1527-1528.

13. Cooper VL, Hainsworth R. Effects of dietary salt on orthostatic tolerance, blood pressure and baroreceptor sensitivity in patients with syncope. Clin Auton Res 2002; 12: 236-241.

14. El-Sayed H, Hainsworth R. Salt supplement increases plasma volume and orthostatic tolerance in patients with unexplained syncope. Heart 1996; 75: 134-140.

15. Mtinangi BL, Hainsworth R. Early effects of oral salt on plasma volume, orthostatic tolerance, and baroreceptor sensitivity in patients with syncope. Clin Auton Res 1998; 8: 231-235.

16. Shichiri M, Tanaka H, Takaya R, Tamai H. Efficacy of high sodium intake in a boy with instantaneous orthostatic hypotension. Clin Auton Res 2002; 12: 4750 .

17. Shannon RP, Wei JY, Rosa RM, Epstein FH, Rowe JW. The effect of age and sodium depletion on cardiovascular response to orthostasis. Hypertension 1986; 8: 438-443.
18. Younoszai AK, Franklin WH, Chan DP, Cassidy SC, Allen HD. Oral fluid therapy. A promising treatment for vasodepressor syncope. Arch Pediatr Adolesc Med 1998; 152: 165-168.

19. Bellard E, Fortrat JO, Custaud MA, Victor J, Greenleaf J, Lefthériotis G. Increased hydration alone does not improve orthostatic tolerance in patients with neurocardiogenic syncope. Clin Auton Res 2007; 17: 99-105.

20. Wieling W, Van Lieshout JJ, Hainsworth R. Extracellular fluid volume expansion in patients with posturally related syncope. Clin Auton Res 2002; 12: 242-249.

21. Guzman JC, Armaganijan LV, Morillo CA. Treatment of neurally mediated reflex syncope. Cardiol Clin 2013; 31: 123-129.

22. Claydon VE, Hainsworth R. Salt supplementation improves orthostatic cerebral and peripheral vascular control in patients with syncope. Hypertension 2004; 43: 809-813.

23. Huang BS, Leenen FH. Dietary Na and baroreflex modulation of blood pressure and RSNA in normotensive vs. spontaneously hypertensive rats. Am J Physiol 1994; 266(2 Pt 2): H496-H502. 